Interactive comment on "Thermal and near-infrared sensor for carbon observation Fourier-transform spectrometer-2 (TANSO-FTS-2) on the Greenhouse Gases Observing Satellite-2 (GOSAT-2) during its first year on orbit” by Hiroshi \title{
Suto et al.
}

Hiroshi Suto et al.

suto.hiroshi@jaxa.jp

Received and published: 29 January 2021

Please find our reply-to-referee Dr. Ray Nassar in the supplement. 
Interactive comment 